\title{
Diet and the risk of gastric cancer: review of epidemiological evidence
}

\author{
Shoichiro Tsugane and Shizuka Sasazuki \\ Epidemiology and Prevention Division, Research Center for Cancer Prevention and Screening, National Cancer Center, 5-1-1 Tsukiji, \\ Chuo-ku, Tokyo 104-0045, Japan
}

\begin{abstract}
There are geographic and ethnic differences in the incidence of gastric cancer around the world as well as with its trends for each population over time. The incidence patterns observed among immigrants change according to where they live. All of these factors serve to indicate the close association of gastric cancer with modifiable factors such as diet. This review presents epidemiological evidence on the association between dietary factors and gastric cancer based on previous systematic reviews and subsequent updates. Infection with Helicobacter pylori is a strong and established risk factor of gastric cancer but is not a sufficient cause for its development. Substantial evidence from ecological, case-control, and cohort studies strongly suggests that the risk may be increased with a high intake of various traditional salt-preserved foods and salt per se and decreased with a high intake of fruit and vegetables, particularly fruit. However, it remains unclear which constituents in fruit and vegetables play a significant role in gastric cancer prevention. Among them, vitamin $\mathbf{C}$ is a plausible candidate supported by a relatively large body of epidemiological evidence. Consumption of green tea is possibly associated with a decreased risk of gastric cancer, although the protective effects have been, for the most part, identified in Japanese women, most of whom are nonsmokers. In contrast, processed meat and $N$-nitroso compounds may be positively associated with the risk of gastric cancer. In conclusion, dietary modification by reducing salt and salted food intake, as well as by increasing intake of fruit and vitamin $C$, represents a practical strategy to prevent gastric cancer.
\end{abstract}

Key words Stomach neoplasms - Diet - Salt - Helicobacter pylori $\cdot$ Fruit and vegetables $\cdot$ Epidemiological studies

Offprint requests to: $\mathrm{S}$. Tsugane

Received: March 8, 2007 / Accepted: April 15, 2007

\section{Introduction}

In 2000, gastric cancer was the second most frequent cause of cancer death worldwide and the fourth most common cancer, with an estimated 650000 deaths and 880000 new cases per year. Almost two-thirds of these new cases occurred in developing countries [1]. In Japan, gastric cancer accounted for 51000 deaths in 2004, or $16 \%$ of all cancer deaths (Vital Statistics: Ministry of Health, Labor and Welfare, Japan); a total of 103000 new cases were detected in 2000 , or $19 \%$ of all incident cancers [2]. The prevention of gastric cancer therefore represents one of the most important aspects of any cancer control strategy in Japan and around the world.

There are geographic and ethnic differences in gastric cancer incidence in the world and in its trends for each population with time. The incidence patterns observed among immigrants change according to where they live. These factors indicate the close association of gastric cancer with modifiable factors such as diet. Substantial evidence from ecological, case-control, and cohort studies strongly suggest that the risk of cancer increases with a high intake of various traditional salt-preserved foods as well as salt per se and that this risk could be decreased with a high intake of fruit and vegetables [3,4]. A recent report of a joint World Health Organization (WHO)/Food and Agriculture Organization (FAO) Expert Consultation concluded that salt-preserved food and salt "probably" increase the risk of gastric cancer, whereas fruit and vegetables "probably" decrease the risk [5]. Other established nondietary factors include cigarette smoking [6] and infection with the bacterium Helicobacter pylori (H. pylori) [7]. In addition, there is some evidence that the intake of green tea and vitamin $\mathrm{C}$ is associated with the risk of gastric cancer.

In this review, we present epidemiological evidence for the association between dietary factors and gastric cancer, with particular reference to our recent work as well as related articles. 


\section{Salt-preserved food and salt}

In experimental studies in rats, ingestion of salt is known to cause gastritis and, on co-administration, to enhance the carcinogenic effects of known gastric carcinogens such as $N$-methyl- $N$-nitro- $N$-nitrosoguanidine (MNNG) $[8,9]$. A high salt concentration in the stomach destroys the mucosal barrier and leads to inflammation and damage such as diffuse erosion and degeneration. Furthermore, the induced proliferative change may act to promote the effect of food-derived carcinogens. It is therefore biologically plausible that high salt intake increases the risk of gastric cancer in humans.

\section{Evidence from ecological studies}

There are substantial geographic differences in the incidence of and mortality associated with gastric cancer worldwide, as well as nationwide in Japan. Using data from the INTERSALT study, in which randomly selected 24-h urine samples from 39 populations were sampled from 24 countries $(n=5756)$, Joossens and colleagues analyzed median sodium levels in samples from subjects aged 20-49 years in relation to the national gastric cancer mortality rates [10]. For the 24 countries, the Pearson correlation of gastric cancer mortality with sodium was 0.70 in men and 0.74 in women (both $P<0.001$ ). In an ecological study of 65 rural counties in China, the consumption of salt-preserved vegetables was correlated with gastric cancer mortality ( $r=0.26$ in men, 0.36 in women) [11].

Approximately threefold differences in agestandardized mortality rates have been identified in Japan, with higher rates in Akita and Yamagata prefectures, lower rates in Kyushu district prefectures such as Kagoshima and Miyazaki, and an especially low rate in Okinawa (Vital Statistics: Ministry of Health, Labor and Welfare, Japan). Our ecological study of five selected areas in Japan showed an almost linear correlation between the cumulative mortality rate for gastric cancer in subjects up to 75 years of age and the urinary salt excretion level in 24-h urine samples [12,13].

Both age-adjusted mortality and incidence rates have been decreasing for several decades in Japan. In the United States [14] and Europe [15], gastric cancer used to be one of the most common cancers; however, mortality rates have fallen dramatically over the last 50 years in all Western countries without any specific intervention taken, and gastric cancer is now rare. This worldwide decline in incidence is likely attributable to the spread of refrigeration, the use of which would inversely correlate with salting and other saltbased methods of preservation such as curing and smoking, and with the overall volume of salt in the diet [3].
Studies in migrants offer clues about the relative importance of genetic and environmental factors in the etiology of cancer and are particularly useful when large differences exist between the original and host countries in incidence and lifestyle. Age-adjusted (world population) incidence rates of gastric cancer among Japanese residents in Hawaii (USA) were significantly lower than in Japan among both men and women, whereas rates in São Paulo, Brazil, were relatively similar to those in Japan [16]. These differences in incidence among three Japanese populations suggest that lifestyle changes, mainly dietary, are associated with a decreased risk of gastric cancer depending on the degree of westernization and the individual incidence rate in the respective host country (United States or Brazil).

According to cross-sectional studies of randomly selected Japanese Brazilians in the city of São Paulo (1989) and Japanese living in five prefectures across Japan (1989-1991), the dietary habits of Japanese Brazilians have shifted toward the pattern seen in Western countries [17]. However, Japanese Brazilians still consumed traditional and salted Japanese foods: 15\% of Japanese Brazilian men aged 40-49 years consumed miso soup almost daily, and 4\% consumed pickled vegetables [18]. The degree of westernization was not striking when compared with that of Japanese Americans. For example, only $2 \%$ of Japanese American men aged 45-69 years in Hawaii consumed miso soup almost daily during the 1960s [19]. Furthermore, salt excretion levels in 24-h urine samples among Japan-born male residents aged 40-59 years in São Paulo were $14.0 \mathrm{~g} /$ day for 21 volunteers originally from Iwate, Akita, and Nagasaki prefectures and $8.7 \mathrm{~g} /$ day for 12 volunteers from Okinawa Prefecture (unpublished data). These levels were closely comparable with those seen in each prefecture in Japan.

The substantial decrease in the incidence of gastric cancer among Japanese immigrants in the United States and the minimal decrease among Japanese immigrants in Brazil can be explained on the basis of the manner in which they continue to maintain Japanese dietary habits, which are typically high in salt.

\section{Evidence from case-control studies}

Many but not all case-control studies have found a positive association between gastric cancer and the intake of highly salted foods such as salted fish, cured meat, and salted vegetables or the use of table salt [4]. Several studies have quantitatively estimated total salt intake and found a strong positive association with the risk of gastric cancer, and several others evaluated its association with the intake of salted food such as salted fish and vegetables. In an evaluation performed by the 
World Cancer Research Fund (WCRF) and the American Institute for Cancer Research (AICR) in 1997 [3], 16 case-control studies reported an association between salt or salted food and the risk of gastric cancer. Eight of these estimated overall dietary salt or sodium intake; four showed strong statistically significant increases in risk [odds ratio $(\mathrm{OR})=2.1-5.0$ for the highest intake level], whereas the remaining four showed no substantial association. Six of sixteen studies specifically examined the use of table salt, with three reporting statistically significant increased risks $(\mathrm{OR}=1.6-6.2$ for highest intakes) and two nonsignificant ORs. Several recent case-control studies have also revealed an association between salted food and the risk of gastric cancer [20-23].

\section{Evidence from cohort studies}

Prospective data are scarce, and only two studies were evaluated in the 1997 report by the WCRF and AICR [3]. One reported no association with the intake of table salt or soy sauce, although the second found an association between salted fish intake and an increased risk of gastric cancer in white American men, largely of Scandinavian and German descent [relative risk $(\mathrm{RR})=1.9$ for the highest intake level].

Four more recent studies have also reported this association. Ngoan and colleagues examined 13000 Japanese men and women and identified 116 gastric cancer deaths during a 10-year follow-up. Higher consumption of pickled foods and traditional soups were associated with increased risk, although without statistical significance [24].

The Netherlands Cohort Study examined 120852 men and women and identified 282 gastric cancer cases at a 6.3-year follow-up [25]. Salt intake was measured by calculating the mean daily sodium intake (dietary salt) from 150 food items and by specific questions related to the consumption of salt. The intake of dietary salt and several types of cured meat showed a weak positive association with the risk of gastric cancer.

The Hisayama study examined 2476 men and women and identified 93 gastric cancer cases during a 14-year follow-up [26]. Dietary salt intake from a 70-item food frequency questionnaire was significantly associated with the risk of gastric cancer after considering $H$. pylori infection.

Our population-based prospective study examined a total of 18684 men and 20381 women aged 40-59 years and documented 358 men and 128 women with histologically confirmed gastric cancer during the 12 years of follow-up [27]. After adjustment for potential confounding factors, the category salt intake by quintile was dosedependently associated with the risk of gastric cancer in men (for trend, $P<0.001$ ), whereas no clear trend was seen in women $(P=0.48)$. The weak association between salt intake and gastric cancer in women may have been due to the relatively low validity of the estimated salt intake: the Spearman rank correlation with a 2-day urinary excretion level was only 0.12 for women but 0.38 for men. Although the association was less clear for miso soup, pickled vegetables, and dried fish, the frequency categories of highly salted food (e.g., salted fish roe and salted fish preserves) were strongly associated with gastric cancer risk in both sexes.

These findings imply that either the intake of highly salted food increases the risk of gastric cancer or that it is merely a good marker of a preference for salted food or salt intake in general. An alternative explanation for the strong association between highly salted food and gastric cancer might involve the presence of chemical carcinogens such as $N$-nitroso compounds, which are formed by the reacting nitrate or nitrite during the process of preservation and during digestion in the stomach. A recent meta-analysis based on six prospective and nine case-control studies showed that the consumption of processed meat was associated with an increased risk of gastric cancer [28]. Of note, processed meat often contains chemical carcinogens such as the $\mathrm{N}$-nitroso compounds as well as high amounts of salt.

\section{Salt, salted food intake, and Helicobacter pylori infection}

Infection with $H$. pylori is an established risk factor of, but not a sufficient cause for, the development of gastric cancer $[7,29]$. It is important to elucidate the role salt and salted food play in the causal link between $H$. pylori infection and gastric cancer.

We previously tested the association between lifestyle factors and $H$. pylori infection in a cross-sectional study of 634 men aged 40-49 years selected randomly from five areas in Japan. A total of 474 of the 628 men evaluated were positive for immunoglobulin $\mathrm{G}$ antibody against the bacterium [30]. Intake of pickled vegetables was positively associated with the prevalence of $H$. pylori (ORs against men who consume $<1$ day/ week $=1.19$ for $1-2$ days/week, 1.92 for 3-4 days/week, and 1.90 for 5-7 days/week; for trend, $P=0.02$ ), as was the daily consumption of miso soup [OR against nondaily consumers $=1.60,95 \%$ confidence interval $(\mathrm{CI})=$ 1.03-2.49]. In contrast, occupation, number of siblings, education, smoking, alcohol consumption, and other dietary habits were not significantly associated with the prevalence of infection in this population. Although there are limitations to cross-sectional studies such as this one, the consumption of salted food appears to increase the risk of $H$. pylori infection.

Mucosal damage induced by salt and salted food may increase the possibility of persistent infection with 
H. pylori [31]. Although salted food intake may increase the risk of $H$. pylori infection, salt per se was shown to act synergistically and dose-dependently to promote the development of gastric adenocarcinoma in Mongolian gerbils treated with $N$-methyl- $N$-nitrosourea (MNU) [32,33]. A synergistic exacerbatory effect of $H$. pylori infection and salted food intake has also been reported in case-control studies in Japan [34] and Korea [23].

Infection with $H$. pylori, however, is by itself unlikely to increase the intake of salted food, and $H$. pylori infection therefore cannot act as a confounder in the causal link between salt and salted food intake and gastric cancer. Restricting salt and salted food intake can, at the least, reduce the risk of gastric cancer.

In summary, a large body of evidence from descriptive and analytical epidemiological studies on the association between salt and salted food intake and the risk of gastric cancer indicates that dietary modification to reduce salt and salted food intake is probably protective against gastric cancer even after considering $H$. pylori infection.

\section{Fruit and vegetables}

Fruit and vegetables are rich sources of carotenoids, vitamin $\mathrm{C}$, folate, and phytochemicals, which may have a protective role in the carcinogenesis process. It is likely that modulation of xenobiotic-metabolizing enzymes, in particular phase II enzymes, contributes to this putative preventive mechanism. The mechanisms of antioxidant activity may be also possible.

In 1997, an expert panel assembled by the WCRF and AICR concluded that diets high in fruit and vegetables "convincingly" protect against gastric cancer [3]. This evaluation was based mainly on reports of case-control studies. Since then, however, several cohort studies have reported conflicting results. The joint $\mathrm{WHO} / \mathrm{FAO}$ Expert Consultation in 2003 concluded that fruit and vegetables "probably," but not "convincingly," decrease the risk of gastric cancer [5].

A subsequent report by the International Agency for Research on Cancer (IARC) determined that higher intake of fruit "probably" and higher intake of vegetables "possibly" reduce the risk of gastric cancer [35]. For fruit, the association has been evaluated in 10 cohort studies, most of which reported an inverse association with a summary value of $0.85(95 \% \mathrm{CI}=0.77-0.95)$. Inverse associations were more striking in the 28 evaluable case-control studies, with summary ORs of 0.63 (95\% CI $=0.58-0.69)$. For vegetables, the association has been evaluated in five cohort studies, most of which reported RRs below 1.0. However, none of these RR values were statistically significant, and the summary value was 0.94 (95\% CI $=0.84-1.06)$. In contrast, most of the 20 evaluable case-control studies provided statistically significant ORs below 1.0 and a summary value of 0.66 (95\% CI $=0.61-0.71)$. The reason case-control studies were more likely to show an inverse association is not clear, although one explanation might be the recall bias inherent to case-control studies. Furthermore, people with preclinical symptoms of gastric cancer or stomach disorders may change their dietary habits months or years before diagnosis.

A meta-analysis of cohort studies published up to 2004 reported nonsignificant summary estimates (RR for the highest versus the lowest consumption category) of 0.89 (95\% CI $=0.78-1.02)$ for fruit (13 studies) and $0.98(95 \% \mathrm{CI}=0.86-1.13)$ for vegetables (8 studies) [36]. However, the inverse associations became clearer when the studies were limited to those with incidence data (seven studies for fruit: $\mathrm{RR}=0.82,95 \%$ $\mathrm{CI}=0.73-0.93$; five studies for vegetables: $\mathrm{RR}=0.88$, $95 \% \mathrm{CI}=0.69-1.13$ ) and with follow-up periods of 10 years or longer (three studies for fruit: $\mathrm{RR}=0.66,95 \%$ $\mathrm{CI}=0.52-0.83$; two studies for vegetables: $\mathrm{RR}=0.71$, $95 \%$ CI $=0.53-0.94)$.

Subsequent to the evaluation by the IARC [35] and the meta-analysis of Lunet et al. [36], several cohort studies have reported the association with fruit and vegetables. In a study in Sweden (139 gastric cancer cases among 70000 men and women) [37], subjects who consumed 2.5 servings of vegetables or more per day had a hazard ratio of $0.56(95 \% \mathrm{CI}=0.34-0.93)$ compared with those who consumed less than 1.0 serving per day. The respective hazard ratio for fruit consumption was 0.86 (95\% CI $=0.52-1.43)$. In the European Prospective Investigation into Cancer and Nutrition (330 gastric cancer cases among 520000 men and women) [38], a protective role for vegetable intake was observed for the intestinal type of gastric cancer. Citrus fruit consumption may play a role in protection against gastric cardia cancer.

In our cohort study with 400 gastric cancer cases among 40000 men and women, the RR associated with intake 1 day or more per week compared with less than 1 day per week was $0.64(95 \% \mathrm{CI}=0.45-0.92)$ for yellow vegetables, $0.48(95 \% \mathrm{CI}=0.25-0.89)$ for white vegetables, and $0.70(95 \% \mathrm{CI}=0.49-1.00)$ for fruit. $\mathrm{RRs}$ associated with the quintile of total vegetable consumption were 1.00, 0.86, 0.75, 0.90, and 0.75 (for trend, $P=$ $0.17)$. This association became clearer for the differentiated type of gastric cancer, at 1.00, 0.96, 0.78, 0.88, and 0.53 (for trend, $P=0.03$ ). These findings suggest that vegetable and fruit intake, even in relatively low amounts, is associated with a lower risk of gastric cancer.

In summary, consumption of fruit and vegetables, particularly fruit, is probably protective against gastric cancer. Nevertheless, it remains unknown which con- 
stituents in fruit and vegetables play a significant role in gastric cancer prevention.

\section{Vitamin C (ascorbic acid)}

Fruit and vegetables are rich sources of vitamin C. Vitamin $\mathrm{C}$ acts as an antioxidant and can quench reactive oxygen species produced in the gastric environment [39]; it is also known to inhibit production of carcinogenic $N$-nitroso compound in the stomach [40]. A possible relation between $H$. pylori infection and ascorbic acid is under investigation, as some research has indicated that high-dose vitamin $\mathrm{C}$ is effective in inhibiting H. pylori infection $[41,42]$.

The WCRF/AICR report [3] concluded that high dietary vitamin $\mathrm{C}$ intake probably decreases the risk of gastric cancer. This conclusion is based on 1 of 2 cohort studies and 12 of 13 case-control studies showing inverse associations between dietary vitamin $\mathrm{C}$ intake and the risk of gastric cancer. In addition, one prospective study showed that baseline plasma vitamin C levels among subjects who died from gastric cancer $(n=20)$ was $20 \%$ lower than those who remained cancer-free $(n=2421)$ during more than 12 years of follow-up [43].

Since then a limited number of prospective studies have directly tested the association between dietary intake or blood level of vitamin $\mathrm{C}$ and the risk of gastric cancer. Dietary intake from comprehensive food frequency questionnaires was inversely associated with the risk of noncardia gastric cancer $(n=179)$ during a median follow-up of 12 years in Finnish male smokers [44] and with the risk of gastric cancer $(n=282)$ over 6.3 years in a Dutch cohort [45], with an inverse association, albeit diminished, in the latter study after excluding cases diagnosed within 2 years of baseline.

A nested case-control study in the European Prospective Investigation into Cancer and Nutrition (EPIC) compared the levels of dietary and prediagnostic plasma vitamin $\mathrm{C}$ between 215 gastric cancer cases and 416 matched controls [46]. No association with gastric cancer risk was observed for dietary vitamin $\mathrm{C}$, whereas an inverse association was seen in the highest versus lowest quartile of plasma vitamin $\mathrm{C}(\mathrm{OR}=0.55,95 \% \mathrm{CI}=$ 0.31-0.97; for trend, $P=0.04$ ). This inverse association was more pronounced in subjects consuming high levels of red and processed meat, a factor that may increase endogenous $N$-nitroso compound production. In a nested case-control study in a cohort of 20000 Chinese men (191 cases and 570 matched controls), increased serum levels of vitamin $\mathrm{C}$ were significantly associated with a reduced risk of gastric cancer among never-smoker and non-heavy-alcohol drinking men (for trend, $P=0.02$ ) [47].
The use of vitamin $\mathrm{C}$ supplements was inversely and nonsignificantly associated with the risk of gastric cancer mortality $(n=1725 ; \mathrm{RR}=0.83,95 \% \mathrm{CI}=0.68-1.01)$ among a million U.S. adults followed for 16 years (Cancer Prevention Study II) [48]. However, the decrease in risk was observed among users of shorter duration only, not among those of longer duration.

A nutritional intervention trial in Linxian, China, showed that supplementation with $\beta$-carotene $(15 \mathrm{mg})$, $\alpha$-tocopherol (30 mg), and selenium $(50 \mu \mathrm{g})$ reduced the risk of gastric cancer mortality by approximately $20 \%$ after 5.25 years [49]. Other micronutrients, including vitamin C $(120 \mathrm{mg})$, however, were associated with no reduction. In contrast, a chemoprevention trial of gastric dysplasia in Colombia in which vitamin $\mathrm{C}$ supplementation at $2 \mathrm{~g}$ /day for 6 years was tested as one of three treatment regimens showed that vitamin $\mathrm{C}$ as well as $30 \mathrm{mg} \beta$-carotene and anti-H. pylori therapy increased the regression rate of gastric precancerous lesions [50].

In our population-based, double-blind, randomized controlled trial to examine the effect of 5 years of vitamin C supplementation, a statistically significant difference was seen between the high-dose group $(500 \mathrm{mg} /$ day $)$ and the low-dose group $(50 \mathrm{mg} /$ day $)(P=0.046)$ in the change in the pepsinogen I/pepsinogen II ratio, a marker of mucosal atrophic change in the stomach. This finding suggests that vitamin C supplementation may protect against the progression of gastric mucosal atrophy [51].

In summary, when the relatively consistent epidemiological evidence and biological plausibility are considered, dietary or supplemental vitamin C intake probably decreases the risk of gastric cancer.

\section{Green tea and other beverages}

\section{Green tea}

Green tea contains polyphenols, more commonly known as catechins. Major green tea catechins include (-)epigallocatechin-3-gallate (EGCG), (-)-epigallocatechin (EGC), (-)-epicatechin-3-gallate (ECG), and (-)-epicatechin (EC). Antioxidant activities and the ability to inhibit the nitrosation of polyphenols have been isolated from green tea in both in vitro and in vivo studies [52-54]. In addition, recent research has proposed many other possible mechanisms for the cancerinhibitory effects of green tea, including modulation of signal transduction pathways, leading to the inhibition of cell proliferation and transformation, induction of apoptosis and cell cycle arrest, and inhibition of tumor invasion and angiogenesis [55-57].

The WCRF/AICR report [3] included five casecontrol studies investigating the relation between green 
tea intake and gastric cancer risk. Among these studies, four suggested a protective effect. Although a Japanese study showed a significant decrease in risk only among those consuming 10 cups or more per day, a Chinese study identified a clear dose relation. In contrast, a casecontrol study in Japan observed no material associations for green tea. Based on these findings, the WCRF/AICR report concluded that high consumption of green tea "possibly" decreases the risk of gastric cancer. More recent case-control studies have also shown a reduction in the risk with green tea intake, most with statistical significance [58-61].

In contrast to laboratory studies and most casecontrol studies, however, all but one recent cohort study have shown no protective effect of green tea for gastric cancer [62-65]. In a population-based prospective study conducted in Miyagi Prefecture in northern Japan, the RRs associated with drinking one or two, three or four, and five or more cups of green tea per day, as compared with less than one cup per day, were $1.3(95 \% \mathrm{CI}=0.8-1.9), 1.2(95 \% \mathrm{CI}=0.8-1.8)$, and 1.5 $(95 \%$ CI $=1.0-2.1)$, respectively, in men (for trend, $P$ $=0.03)$, and $0.8(95 \% \mathrm{CI}=0.5-1.5), 0.7$ (95\% CI $=$ $0.4-1.3)$, and $0.8(95 \% \mathrm{CI}=0.5-1.3)$, respectively, in women (for trend, $P=0.46$ ) [62]. In a nationwide multicenter prospective study, no inverse association was found between green tea consumption and gastric cancer death, with the risks associated with drinking 1 or 2,3 or $4,5-9$, and $\geq 10$ cups of green tea per day, relative to those of drinking less than 1 cup per day, of 1.6 $(95 \% \mathrm{CI}=0.9-2.9), 1.1(95 \% \mathrm{CI}=0.6-1.9), 1.0(95 \%$ $\mathrm{CI}=0.5-2.0)$, and $1.0(95 \% \mathrm{CI}=0.5-2.0)$, respectively, in men (for trend, $P=0.669)$, and $1.1(95 \% \mathrm{CI}=0.5-$ 2.5), 1.0 (95\% CI $=0.5-2.5), 0.8(95 \% \mathrm{CI}=0.4-1.6)$, and $0.8(95 \% \mathrm{CI}=0.3-2.1)$, respectively, in women (for trend, $P=0.448$ ) [63]. Furthermore, green tea consumption was virtually unrelated to the incidence of any cancer, including gastric cancer, in a follow-up study of atomic bomb survivors in Hiroshima and Nagasaki [64].

In our cohort study, among 73000 subjects with 890 gastric cancers, although no association between green tea consumption and gastric cancer was observed among men, decreased risk was suggested in women, with RRs and $95 \%$ CI for one or two, three or four, and five or more cups per day compared to less than one cup per day of 0.85 (95\% CI $=0.53-1.38), 1.04$ (95\% CI $=0.68-$ 1.58 ), and 0.67 (95\% CI $=0.43-1.04$ ), respectively (for trend, $P=0.08$ ) [65]. This association was further strengthened when cancer was restricted to the distal portion, with a RR of $0.51(95 \% \mathrm{CI}=0.30-0.86)$ for consumption of five or more cups of green tea (for trend, $P=0.01)$. These results are consistent with those of previous prospective studies in that decreased risk was more apparent in women.
In summary, consumption of green tea is possibly associated with decreased risk of gastric cancer, especially in Japanese women, most of whom are nonsmokers. Further prospective studies with detailed information are needed to clarify the role of green tea on gastric carcinogenesis.

\section{Black tea and coffee}

Although catechin levels in black tea are only about $30 \%$ of those in green tea, the inhibitory activity of black tea against tumorigenesis has been shown to be comparable to that of green tea in several animal models [66]. Most of three prospective studies and 12 casecontrol studies showed no association with the risk of gastric cancer. Thus, the WCRF/AICR found that high consumption of black tea "probably" has no association with the risk of gastric cancer [3]. Only one prospective study in Japan has been conducted since then, and it showed a nonsignificant increase in the risk of gastric cancer among women who drink black tea more than several times per week [67]. It is possible that lifestyle, which was not investigated in this study, was associated with the increased risk of gastric cancer. Thus, the evidence is less clear for black tea than for green tea.

Caffeine, kahweol, and cafestol in coffee may contribute to a protective effect against cancer [68]. Based on the evaluation of two prospective and eight casecontrol studies, which showed no statistically significant association between coffee consumption and gastric cancer, the WCRF/AICR concluded that high consumption of coffee "probably" has no relation with the risk of gastric cancer [3]. In a more recent systematic review and meta-analysis of 23 studies ( 7 cohort studies and 16 case-control studies) [68], coffee intake showed no effect on gastric cancer when all studies were combined $(\mathrm{OR}=0.97,95 \% \mathrm{CI}=0.86-1.09)$, with a combined risk estimate of $1.02(95 \% \mathrm{CI}=0.76-1.37)$ for cohort studies, $0.90(95 \% \mathrm{CI}=0.70-1.15)$ for population-based casecontrol studies, and 0.97 (95\% CI $=0.83-1.13$ ) for hospital-based case-control studies. Notwithstanding that risk estimates differed significantly according to country of origin, with North American studies presenting a significantly higher risk, this meta-analysis showed no overall effect of coffee consumption on gastric cancer risk.

In summary, there is no apparent evidence that black tea or coffee consumption has any effect on the risk of gastric cancer.

\section{Conclusions}

Although the eradication of $H$. pylori may be a promising strategy for gastric cancer prevention, most middle- 
aged and elderly Japanese are already infected with this pathogen [30]. The worldwide decrease in the ageadjusted incidence of gastric cancer over the last 50 years occurred without any intentional $H$. pylori infection eradication program. Rather, and perhaps more importantly, it can be related to decreased intake of salt and salted food and increased intake of fresh fruit and vegetables, which arose subsequent to the mass availability of refrigeration. Dietary modification to reduce salt and salted food intake and to increase intake of fruit, particularly vitamin $\mathrm{C}$ - as well as quitting smoking - represents an effective, practical, low-cost means of preventing gastric cancer.

Acknowledgments This study was supported by Grantsin-aid for Cancer Research, for the Third Term Comprehensive Ten-Year Strategy for Cancer Control, and for Risk Analysis Research on Food and Pharmaceuticals from the Ministry of Health, Labor and Welfare of Japan, and for Scientific Research from the Ministry of Education, Culture, Sports, Science, and Technology.

\section{References}

1. Stewart BW, Kleihues P, editors. World Cancer Report. Lyon: IARC Press; 2003.

2. Marugame T, Kamo K, Katanoda K, Ajiki W, Sobue T. Japan Cancer Surveillance Research Group. Cancer incidence and incidence rates in Japan in 2000: estimates based on data from 11 population-based cancer registries. Jpn J Clin Oncol 2006; 36:668-75.

3. World Cancer Research Fund, American Institute for Cancer Research. Food, nutrition and the prevention of cancer: a global perspective. Washington, DC: American Institute for Cancer Research; 1997.

4. Kono S, Hirohata T. Nutrition and stomach cancer. Cancer Causes Control 1996;7:41-55.

5. World Health Organization. Diet, nutrition and the prevention of chronic diseases. WHO technical report series 916. Geneva: World Health Organization; 2003.

6. International Agency for Research on Cancer. Tobacco smoking and tobacco smoke. IARC monographs on the evaluation of the carcinogenic risk of chemicals to humans. Vol. 83. Lyon: International Agency for Research on Cancer; 2004.

7. International Agency for Research on Cancer. Schistosomes, liver flukes, and Helicobacter pylori. IARC monographs on the evaluation of carcinogenic risks to humans. Vol. 61. Lyon: International Agency for Research on Cancer; 1994.

8. Tatematsu M, Takahashi M, Fukushima S, Hananouchi M, Shirai $\mathrm{T}$. Effects in rats of salt on experimental gastric cancers induced by $N$-methyl- $N$-nitro- $N$-nitrosoguanidine or 4-nitroquinoline-1oxide. J Natl Cancer Inst 1975;55:101-6.

9. Takahashi M, Hasegawa R. Enhancing effects of dietary salt on both initiation and promotion stages of rat gastric carcinogenesis. Princess Takamatsu Symp 1985;16:169-82.

10. Joossens JV, Hill MJ, Elliott P, Stamler R, Lesaffre E, Dyer A, et al. Dietary salt, nitrate and stomach cancer mortality in 24 countries: European Cancer Prevention (ECP) and the INTERSALT Cooperative Research Group. Int J Epidemiol 1996;25: 494-504.
11. Kneller RW, Guo WD, Hsing AW, Chen JS, Blot WJ, Li JY, et al. Risk factors for stomach cancer in sixty-five Chinese counties. Cancer Epidemiol Biomarkers Prev 1992;1:113-18.

12. Tsugane S, Gey F, Ichinowatari Y, Miyajima Y, Ishibashi T, Matsushima S, et al. Cross-sectional epidemiologic study for assessing cancer risks at the population level. I. Study design and participation rate. J Epidemiol 1992;2:75-81.

13. Tsugane S, Gey F, Ichinowatari Y, Miyajima Y, Ishibashi T, Matsushima S, et al. Cross-sectional epidemiologic study for assessing cancer risks at the population level. II. Baseline data and correlation analysis. J Epidemiol 1992;2:83-9.

14. National Cancer Institute. Cancer rates and risks: cancer mortality in the United States, 1950-91. Changing pattern for major cancers. http://seer.cancer.gov/publications/raterisk/index. $\mathrm{html} /$.

15. La Vecchia C, Franceschi S, Levi F. Epidemiological research on cancer with a focus on Europe. Eur J Cancer Prev 2003;12: $5-14$.

16. Tsugane S, de Souza JM, Costa ML Jr, Mirra AP, Gotlieb SL, Laurenti R, et al. Cancer incidence rates among Japanese immigrants in the city of Sao Paulo, Brazil, 1969-78. Cancer Causes Control 1990;1:189-93.

17. Tsugane S, Hamada GS, Souza JM, Gotlieb SLD, Takashima Y, Todoriki H, et al. Lifestyle and health related factors among randomly selected Japanese residents in the city of Sao Paulo, Brazil, and their comparisons with Japanese in Japan. J Epidemiol 1994:4:37-46.

18. Tsugane S, Hamada GS, Karita K, Tsubono Y, Laurenti R. Cancer patterns and lifestyle among Japanese immigrants and their descendants in the city of Sao Paulo, Brazil. Gann Monogr Cancer Res 1996;44:43-50.

19. Severson RK, Nomura AM, Grove JS, Stemmermann GN. A prospective study of demographics, diet, and prostate cancer among men of Japanese ancestry in Hawaii. Cancer Res 1989; 49:1857-60.

20. Ye WM, Yi YN, Luo RX, Zhou TS, Lin RT, Chen GD. Diet and gastric cancer: a case-control study in Fujian Province, China. World J Gastroenterol 1998;4:516-18.

21. Ward MH, Lopez-Carrillo L. Dietary factors and the risk of gastric cancer in Mexico City. Am J Epidemiol 1999;149: 925-32.

22. Kim HJ, Chang WK, Kim MK, Lee SS, Choi BY. Dietary factors and gastric cancer in Korea: a case-control study. Int J Cancer 2002;97:531-5.

23. Lee SA, Kang D, Shim KN, Choe JW, Hong WS, Choi H. Effect of diet and Helicobacter pylori infection to the risk of early gastric cancer. J Epidemiol 2003;13:162-8.

24. Ngoan LT, Mizoue T, Fujino Y, Tokui N, Yoshimura T. Dietary factors and stomach cancer mortality. Br J Cancer 2002;87: $37-42$.

25. Van den Brandt PA, Botterweck AA, Goldbohm RA. Salt intake, cured meat consumption, refrigerator use and stomach cancer incidence: a prospective cohort study (Netherlands). Cancer Causes Control 2003;14:427-38.

26. Shikata K, Kiyohara Y, Kubo M, Yonemoto K, Ninomiya T, Shirota $\mathrm{T}$, et al. A prospective study of dietary salt intake and gastric cancer incidence in a defined Japanese population: the Hisayama study. Int J Cancer 2006;119:196-201.

27. Tsugane S, Sasazuki S, Kobayashi M, Sasaki S, JPHC Study Group. Salt and salted food intake and subsequent risk of gastric cancer among middle-aged Japanese men and women. Br J Cancer 2004;90:128-34.

28. Larsson SC, Orsini N, Wolk A. Processed meat consumption and stomach cancer risk: a meta-analysis. J Natl Cancer Inst 2006;98: 1078-87.

29. Helicobacter and Cancer Collaborative Group. Gastric cancer and Helicobacter pylori: a combined analysis of 12 case-control studies nested within prospective cohorts. Gut 2001;49:34753. 
30. Tsugane S, Tei Y, Takahashi T, Watanabe S, Sugano K. Salty food intake and risk of Helicobacter pylori infection. Jpn J Cancer Res 1994:85:474-8.

31. Fox JG, Dangler CA, Taylor NS, King A, Koh TJ, Wang TC. High-salt diet induces gastric epithelial hyperplasia and parietal cell loss, and enhances Helicobacter pylori colonization in C57BL/6 mice. Cancer Res 1999;59:4823-8.

32. Nozaki K, Shimizu N, Inada K, Tsukamoto T, Inoue M, Kumagai $\mathrm{T}$, et al. Synergistic promoting effects of Helicobacter pylori infection and high-salt diet on gastric carcinogenesis in Mongolian gerbils. Jpn J Cancer Res 2002;93:1083-9.

33. Kato S, Tsukamoto T, Mizoshita T, Tanaka H, Kumagai T, Ota $\mathrm{H}$, et al. High salt diets dose-dependently promote gastric chemical carcinogenesis in Helicobacter pylori-infected Mongolian gerbils associated with a shift in mucin production from glandular to surface mucous cells. Int J Cancer 2006;119:1558-66.

34. Machida-Montani A, Sasazuki S, Inoue M, Natsukawa S, Shaura $\mathrm{K}$, Koizumi Y, et al. Association of Helicobacter pylori infection and environmental factors in non-cardia gastric cancer in Japan. Gastric Cancer 2004;7:46-53.

35. International Agency for Research on Cancer. Fruit and vegetables. IARC handbooks of cancer prevention. Vol. 8. Lyon: IARC; 2003.

36. Lunet N, Lacerda-Vieira A, Barros H. Fruit and vegetables consumption and gastric cancer: a systematic review and metaanalysis of cohort studies. Nutr Cancer 2005;53:1-10.

37. Larsson SC, Bergkvist L, Wolk A. Fruit and vegetable consumption and incidence of gastric cancer: a prospective study. Cancer Epidemiol Biomarkers Prev 2006;15:1998-2001.

38. Gonzalez CA, Pera G, Agudo A, Bueno-de-Mesquita HB, Ceroti $\mathrm{M}$, Boeing $\mathrm{H}$, et al. Fruit and vegetable intake and the risk of stomach and oesophagus adenocarcinoma in the European Prospective Investigation into Cancer and Nutrition (EPICEURGAST). Int J Cancer. 2006;118:2559-66.

39. Drake IM, Davies MJ, Mapstone NP, Dixon MF, Schorah CJ, White KL, et al. Ascorbic acid may protect against human gastric cancer by scavenging mucosal oxygen radicals. Carcinogenesis 1996;17:559-62.

40. Tannenbaum SR, Wishnok JS, Leaf CD. Inhibition of nitrosamine formation by ascorbic acid. Am J Clin Nutr 1991;53: 247S-50S.

41. Zhang HM, Wakisaka N, Maeda O, Yamamoto T. Vitamin C inhibits the growth of a bacterial risk factor for gastric carcinoma: Helicobacter pylori. Cancer 1997;80:1897-903.

42. Jarosz M, Dzieniszewski J, Ufniarz ED, Wartanowicz M, Ziemlanski S, Reed PI. Effects of high dose vitamin C treatment on Helicobacter pylori infection and total vitamin C concentration in gastric juice. Eur J Cancer Prev 1998;7:449-54.

43. Stahelin HB, Gey KF, Eichholzer M, Ludin E, Bernasconi F, Thurneysen J, et al. Plasma antioxidant vitamins and subsequent cancer mortality in the 12-year follow-up of the prospective Basel study. Am J Epidemiol 1991;133:766-75.

44. Nouraie M, Pietinen P, Kamangar F, Dawsey SM, Abnet CC, Albanes D, et al. Fruits, vegetables, and antioxidants and risk of gastric cancer among male smokers. Cancer Epidemiol Biomarkers Prev 2005;14:2087-92.

45. Botterweck AA, van den Brandt PA, Goldbohm RA. Vitamins, carotenoids, dietary fiber, and the risk of gastric carcinoma: results from a prospective study after 6.3 years of follow-up. Cancer 2000;88:737-48.

46. Jenab M, Riboli E, Ferrari P, Sabate J, Slimani N, Norat T, et al. Plasma and dietary vitamin $\mathrm{C}$ levels and risk of gastric cancer in the European Prospective Investigation into Cancer and Nutrition (EPIC-EURGAST). Carcinogenesis 2006;27: 2250-7.

47. Yuan JM, Ross RK, Gao YT, Qu YH, Chu XD, Yu MC. Prediagnostic levels of serum micronutrients in relation to risk of gastric cancer in Shanghai, China. Cancer Epidemiol Biomarkers Prev 2004;13:1772-80.
48. Jacobs EJ, Connell CJ, McCullough ML, Chao A, Jonas CR, Rodriguez C, et al. Vitamin C, vitamin E, and multivitamin supplement use and stomach cancer mortality in the Cancer Prevention Study II cohort. Cancer Epidemiol Biomarkers Prev 2002; 11:35-41.

49. Blot WJ, Li JY, Taylor PR, Guo W, Dawsey S, Wang GQ, et al. Nutrition intervention trials in Linxian, China: supplementation with specific vitamin/mineral combinations, cancer incidence, and disease-specific mortality in the general population. J Natl Cancer Inst 1993;85:1483-92.

50. Correa P, Fontham ET, Bravo JC, Bravo LE, Ruiz B, Zarama G, et al. Chemoprevention of gastric dysplasia: randomized trial of antioxidant supplements and anti-Helicobacter pylori therapy. J Natl Cancer Inst 2000;92:1881-8.

51. Sasazuki S, Sasaki S, Tsubono Y, Okubo S, Hayashi M, Kakizoe $\mathrm{T}$, et al. The effect of 5-year vitamin C supplementation on serum pepsinogen level and Helicobacter pylori infection. Cancer Sci 2003;94:378-82.

52. Wang ZY, Cheng SJ, Zhou ZC, Athar M, Khan WA, Bickers DR, et al. Antimutagenic activity of green tea polyphenols. Mutat Res 1989;223:273-85.

53. Xu Y, Ho CT, Amin SG, Han C, Chung FL. Inhibition of tobaccospecific nitrosamine-induced lung tumorigenesis in $\mathrm{A} / \mathrm{J}$ mice by green tea and its major polyphenols as antioxidants. Cancer Res 1992;52:3875-9.

54. Wang ZY, Hong JY, Huang MT, Reuhl KR, Conney AH, Yang CS. Inhibition of $N$-nitrosodiethylamine and 4-(methylnitrosamino)-1-(3-pyridyl)-1-butanone-induced tumorigenesis in $\mathrm{A} / \mathrm{J}$ mice by green tea and black tea. Cancer Res 1992;52:1943-7.

55. Yang CS, Maliakal P, Meng X. Inhibition of carcinogenesis by tea. Annu Rev Pharmacol Toxicol 2002;42:25-54.

56. Moyers SB, Kumar NB. Green tea polyphenols and cancer chemoprevention: multiple mechanisms and endpoints for phase II trials. Nutr Rev 2004;62:204-11.

57. Mukhtar H, Ahmad N. Tea polyphenols: prevention of cancer and optimizing health. Am J Clin Nutr 2000;71(suppl):698S-702S.

58. Ji BT, Chow WH, Yang G, McLaughlin JK, Gao RN, Zheng W, et al. The influence of cigarette smoking, alcohol, and green tea consumption on the risk of carcinoma of the cardia and distal stomach in Shanghai, China. Cancer 1996;77:2449-57.

59. Inoue M, Tajima K, Hirose K, Hamajima N, Takezaki T, Kuroishi $\mathrm{T}$, et al. Tea and coffee consumption and the risk of digestive tract cancers: data from a comparative case-referent study in Japan. Cancer Causes Control 1998;9:209-16.

60. Setiawan VW, Zhang ZF, Yu GP, Lu QY, Li YL, Lu ML, et al. Protective effect of green tea on the risks of chronic gastritis and stomach cancer. Int J Cancer 2001;92:600-4.

61. Takezaki T, Gao CM, Wu JZ, Ding JH, Liu YT, Zhang Y, et al. Dietary protective and risk factors for esophageal and stomach cancers in a low-epidemic area for stomach cancer in Jiangsu Provance, China: comparison with those in a high-epidemic area. Jpn J Cancer Res 2001;92:1157-65.

62. Tsubono Y, Nishino Y, Komatsu S, Hsieh CC, Kanemura S, Tsuji I, et al. Green tea and the risk of gastric cancer in Japan. N Engl J Med 2001;344:632-6.

63. Hoshiyama Y, Kawaguchi T, Miura Y, Mizoue T, Tokui N, Yatsuya $\mathrm{H}$, et al. A prospective study of stomach cancer death in relation to green tea consumption in Japan. Br J Cancer 2002;87: 309-13.

64. Nagano J, Kono S, Preston DL, Mabuchi K. A prospective study of green tea consumption and cancer incidence, Hiroshima and Nagasaki, Japan. Cancer Causes Control 2001;15:501-8.

65. Sasazuki S, Inoue M, Hanaoka T, Yamamoto S, Sobue T, Tsugane S. Green tea consumption and subsequent risk of gastric cancer by subsite: the JPHC study. Cancer Causes Control 2004; 15:483-91.

66. Yang CS, Lee MJ, Chen L, Yang GY. Polyphenols as inhibitors of carcinogenesis. Environ Health Perspect 1997;105(suppl 4): 971-6. 
67. Khan MMH, Goto R, Kobayashi K, Suzumura S, Nagata Y, Sonoda T, et al. Dietary habits and cancer mortality among middle aged and older Japanese living in Hokkaido, Japan by cancer site and sex. Asian Pac J Cancer Prev 2004;5:58-65.

68. Botelho F, Lunet N, Barros H. Coffee and gastric cancer: systematic review and meta-analysis. Cad Saude Publica 2006;22: 889-900. 